\title{
SuRface Microstructuring OF PES FABRICS BY UV LASER IRRADIATION
}

\author{
VRINCEANU, N.; COMAN, D.; SANDU, I. \& GRIGORIU, A.
}

Abstract: In this study, laser was used to treat PES fabrics. The effects of laser treatment on the surface morphology of the fibres were characterized using scanning electron microscopy (SEM) and atomic force microscopy (AFM). The microscopic evaluation revealed the changes in the surface morphology of the fibres caused by laser treatment. By surface microstructuring, the treatment achieved can be considered an intelligent solution for the improvement of surface properties of textile materials.

Key words: microstructuring, UV excimer laser irradiation, PES fabrics, morphology
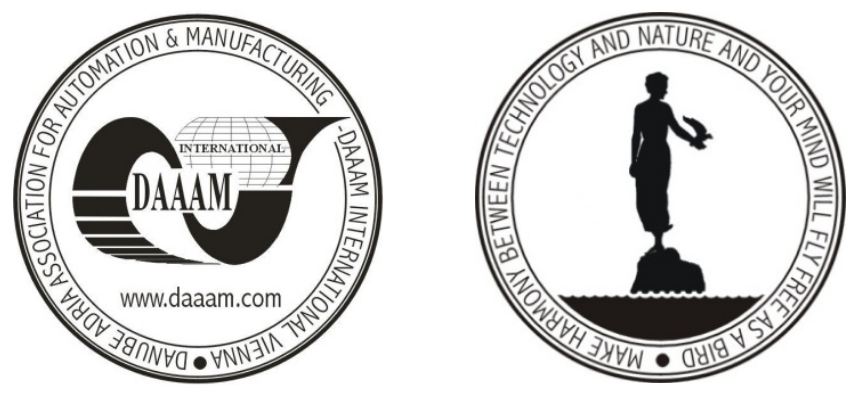

Authors' data: Univ. Teacher Assistant Dipl.-Ing. Dr. Sc. Vrinceanu, N[arcisa]*; Univ. Associate Professor Dipl.-Ing. Dr. Sc. Coman, D[iana]*; Univ. Full Professor Dr. Sc. Sandu I[on] ${ }^{* * *}$; Univ. Full Professor Dipl.-Ing. Dr. Sc. Grigoriu A[urelia]**, *“Lucian Blaga“ University, „Hermann Oberth“ Engineering Faculty, Ion Ratiu 5, 550025, Sibiu, Romania, ** "Gh. Asachi“ Technical University, Faculty of Textile, Leather Engineering and Industrial Management, D. Mangeron 53, 700050, Iasi, Romania, *** „Al. I. Cuza“ University of Iasi, Faculty of History, ARHEOINVEST Platform, Bulevardul Carol I, 11, Iasi, Romania, narcisa.vrinceanu@ulbsibiu.ro,

diana.coman@ulbsibiu.ro,sandu_i03@yahoo.com,augrigor@tex.tuiasi.ro

This Publication has to be referred as: Vrinceanu, N[arcisa]; Coman, D[iana]; Sandu I[on] \& Grigoriu A[urelia] (2009). Surface Microstructuring of PES Fabrics by UV Laser Irridation, Chapter 59 in DAAAM International Scientific Book 2009, pp. 613-620, B. Katalinic (Ed.), Published by DAAAM International, ISBN 978-3901509-69-8, ISSN 1726-9687, Vienna, Austria

DOI: $10.2507 /$ daaam.scibook.2009.59 